Virginia Commonwealth University VCU Scholars Compass

\title{
Determination of the absolute internal quantum efficiency of photoluminescence in GaN co-doped with $\mathrm{Si}$ and $\mathrm{Zn}$
}

\author{
M. A. Reshchikov \\ Virginia Commonwealth University, mreshchikov@vcu.edu \\ M. Foussekis \\ Virginia Commonwealth University \\ J. D. McNamara \\ VirginiaCommonwealth University, mcnamarajd@vcu.edu \\ See next page for additional authors
}

Follow this and additional works at: http://scholarscompass.vcu.edu/phys_pubs

Part of the Physics Commons

Reshchikov, M. A., Foussekis, M., \& McNamara, J. D., et al. Determination of the absolute internal quantum efficiency of photoluminescence in GaN co-doped with Si and Zn. Journal of Applied Physics, 111, 073106 (2012). Copyright (C) 2012 American Institute of Physics.

\section{Downloaded from}

http://scholarscompass.vcu.edu/phys_pubs/124

This Article is brought to you for free and open access by the Dept. of Physics at VCU Scholars Compass. It has been accepted for inclusion in Physics Publications by an authorized administrator of VCU Scholars Compass. For more information, please contact libcompass@vcu.edu. 
Authors

M. A. Reshchikov, M. Foussekis, J. D. McNamara, A. Behrends, A. Bakin, and A. Waag 


\title{
Determination of the absolute internal quantum efficiency of photoluminescence in GaN co-doped with Si and Zn
}

\author{
M. A. Reshchikov, ${ }^{1, a)}$ M. Foussekis, ${ }^{1}$ J. D. McNamara, ${ }^{1}$ A. Behrends, ${ }^{2}$ A. Bakin, ${ }^{2}$ \\ and A. Waag ${ }^{2}$ \\ ${ }^{1}$ Physics Department, Virginia Commonwealth University, Richmond, Virginia 23284, USA \\ ${ }^{2}$ Institute of Semiconductor Technology, Technische Universität Braunschweig, Braunschweig, Germany
}

(Received 30 January 2012; accepted 26 February 2012; published online 10 April 2012)

\begin{abstract}
The optical properties of high-quality GaN co-doped with silicon and zinc are investigated by using temperature-dependent continuous-wave and time-resolved photoluminescence measurements. The blue luminescence band is related to the $\mathrm{Zn}_{\mathrm{Ga}}$ acceptor in $\mathrm{GaN}: \mathrm{Si}, \mathrm{Zn}$, which exhibits an exceptionally high absolute internal quantum efficiency (IQE). An IQE above $90 \%$ was calculated for several samples having different concentrations of $\mathrm{Zn}$. Accurate and reliable values of the IQE were obtained by using several approaches based on rate equations. The concentrations of the $\mathrm{Zn}_{\mathrm{Ga}}$ acceptors and free electrons were also estimated from the photoluminescence measurements. (C) 2012 American Institute of Physics. [http://dx.doi.org/10.1063/1.3699312]
\end{abstract}

\section{INTRODUCTION}

Over the past three decades, $\mathrm{GaN}$ and other nitrides have attracted great interest as materials for short-wavelength lightemitting diodes and laser diodes. Significant efforts are currently being made to increase the internal quantum efficiency (IQE) in these light-emitting devices. However, from a number of methods commonly used to determine the IQE of electroluminescence or photoluminescence (PL) from GaN-based materials or light-emitting devices, only a few appear to be reliable and provide accurate estimates of the IQE. ${ }^{1}$ Often, the ratio of the room-temperature and low-temperature PL intensities is used as a rough estimate of the relative IQE. There is no consensus in the literature on a reliable method of determining the absolute IQE of the PL in GaN. High values of the absolute IQE (30-70\%) were reported for InGaN/GaN quantum wells. ${ }^{2-5}$ However, assumptions made in these publications require more justification. ${ }^{1}$

In this work, we report on the extremely high absolute IQE of the PL in degenerate $n$-type GaN co-doped with $\mathrm{Si}$ and Zn. In our previous work, ${ }^{6}$ preliminary results were reported on the IQE for one sample (sample 1008). In this work, two groups of GaN:Si,Zn samples (capped and uncapped), as well as GaN:Si samples were studied. The IQE was determined from the analysis of the dependencies of the PL intensity on the excitation intensity and temperature. The absolute IQE of the PL in GaN:Si,Zn exceeds $90 \%$, with the largest contribution coming from the blue luminescence (BL) band. In addition, we demonstrate the versatility of the PL technique as a characterization tool for semiconductors. In particular, the concentrations of free electrons, shallow donors, and $\mathrm{Zn}_{\mathrm{Ga}}$ acceptors in GaN doped with only $\mathrm{Si}$ or co-doped with $\mathrm{Si}$ and $\mathrm{Zn}$ have been determined from PL measurements.

\section{EXPERIMENT}

The eight GaN:Si,Zn samples analyzed in this work (Table I) were grown by metalorganic vapor phase epitaxy

\footnotetext{
a)Electronic mail: mreshchi@vcu.edu.
}

(MOVPE) on $c$-plane sapphire substrates at the Technische Universität Braunschweig (TUBS). Ammonia $\left(\mathrm{NH}_{3}\right)$, trimethylgallium (TMGa), $\mathrm{SiH}_{4}$, and DeZn were used as the sources of N, Ga, Si, and $\mathrm{Zn}$, respectively. For the four samples of group A, a $2 \mu \mathrm{m}$-thick undoped GaN layer was grown, and subsequently a $0.4 \mu$ m-thick $\mathrm{AlGaN}$ insulating barrier was deposited. The growth was finished with a $0.2 \mu$ m-thick $\mathrm{GaN}: \mathrm{Si}, \mathrm{Zn}$ active layer. The four samples of group B had the same structure, but were additionally capped with a $40 \mathrm{~nm}$ thick AlGaN barrier. The DeZn flow rate during the growth of the GaN:Si,Zn layer was varied from 4 to $66 \mu \mathrm{mol} / \mathrm{min}$ for samples in each group to vary the concentration of $\mathrm{Zn}$ acceptors (Table I). Additionally, we studied a control sample which was grown under the same conditions, but with only $\mathrm{Si}$ doping of the active layer (sample 1150); as well as another Si-doped GaN sample grown by hydride vapor phase epitaxy (HVPE) at TDI, Inc. (sample 2015). The concentrations of free electrons in the GaN:Si,Zn layer for the samples in group $\mathrm{A}$ and in the Si-doped GaN samples have been found from the Hall effect measurements (Table I). All of the samples were degenerate, except for sample 2015. The concentration of electrons in the GaN:Si, $\mathrm{Zn}$ samples from group B could not be determined by the Hall effect measurements because of the insulating AlGaN capping layer. However, the electron concentration in these samples was estimated from time-resolved PL measurements to be about $10^{19} \mathrm{~cm}^{-3}$, as will be demonstrated in Sec. III C.

Steady-state PL was excited with a $50 \mathrm{~mW}$ He-Cd laser, dispersed by a 1200 rules $/ \mathrm{mm}$ grating in a $0.3 \mathrm{~m}$ monochromator, and was detected by a cooled photomultiplier tube. By using calibrated neutral density filters and an unfocused laser beam with a diameter of $4 \mathrm{~mm}$, the excitation power density $\left(P_{\text {exc }}\right)$ was varied from $10^{-5}$ to $0.3 \mathrm{~W} / \mathrm{cm}^{2}$. The beam could be focused to a diameter of $0.1-0.2 \mathrm{~mm}$ to obtain an $P_{\text {exc }}$ up to $200 \mathrm{~W} / \mathrm{cm}^{2}$. Time-resolved PL was excited with a pulsed nitrogen laser $(337 \mathrm{~nm})$ and analyzed with an oscilloscope. A closed-cycle optical cryostat and a hightemperature cryostat were employed to achieve sample temperatures of 15-330 K and 295-700 K, respectively. The PL 
TABLE I. Characteristics of GaN samples and PL.

\begin{tabular}{|c|c|c|c|c|c|c|c|c|c|}
\hline Sample & $\begin{array}{l}\text { DeZn flow } \\
(\mu \mathrm{mol} / \mathrm{min})\end{array}$ & Group & $\begin{array}{l}n \text { from Hall } \\
\left(\mathrm{cm}^{-3}\right)\end{array}$ & $\begin{array}{l}n \text { from TR-PL } \\
\quad\left(\mathrm{cm}^{-3}\right)\end{array}$ & $\begin{array}{c}N_{1} \equiv N_{Z n} \text { by method } 1 \\
\left(\mathrm{~cm}^{-3}\right)\end{array}$ & $\begin{array}{c}N_{1} \equiv N_{Z n} \text { by method } 2 \\
\left(\mathrm{~cm}^{-3}\right)\end{array}$ & $\begin{array}{c}\tau_{1} \text { at } 200 \mathrm{~K} \\
(\mu \mathrm{s})\end{array}$ & $\begin{array}{l}T_{0} \\
(\mathrm{~K})\end{array}$ & $\begin{array}{c}E_{A 1} \\
(\mathrm{meV})\end{array}$ \\
\hline 1143 & 4 & A & $1.03 \times 10^{19}$ & $1.3 \times 10^{19}$ & $7 \times 10^{17}$ & $2.9 \times 10^{17}$ & 0.52 & 289 & 310 \\
\hline 1142 & 10 & A & $1.03 \times 10^{19}$ & $1.2 \times 10^{19}$ & $5 \times 10^{17}$ & $6.7 \times 10^{17}$ & 0.55 & 296 & 310 \\
\hline 1141 & 33 & A & $1.14 \times 10^{19}$ & $9.5 \times 10^{18}$ & $1.5 \times 10^{18}$ & $1.3 \times 10^{18}$ & 0.70 & 313 & 340 \\
\hline 1140 & 66 & A & $0.75 \times 10^{19}$ & $4.5 \times 10^{18}$ & $3 \times 10^{18}$ & $4.7 \times 10^{18}$ & 1.48 & 305 & 290 \\
\hline 1010 & 4 & B & - & $1.8 \times 10^{19}$ & $1 \times 10^{18}$ & $5.0 \times 10^{17}$ & 0.37 & 289 & 320 \\
\hline 1009 & 10 & B & - & $1.8 \times 10^{19}$ & $2 \times 10^{18}$ & $1.1 \times 10^{18}$ & 0.38 & 294 & 315 \\
\hline 1008 & 33 & B & - & $1.4 \times 10^{19}$ & $5 \times 10^{18}$ & $2.0 \times 10^{18}$ & 0.47 & 300 & 300 \\
\hline 997 & 66 & B & - & $5.2 \times 10^{18}$ & $1 \times 10^{19}$ & $5.3 \times 10^{18}$ & 1.27 & 311 & 345 \\
\hline 1150 & - & - & $1.1 \times 10^{19}$ & - & - & $2 \times 10^{13}$ & - & 250 & 345 \\
\hline $2015^{\mathrm{a}}$ & - & - & $2 \times 10^{17}$ & $6 \times 10^{17}$ & $3 \times 10^{15}$ & - & 15 & 220 & 345 \\
\hline
\end{tabular}

a $5 \mu$ m-thick GaN:Si sample grown by HVPE method at TDI.

spectra were corrected for the response of the optical system by comparing the spectrum of a tungsten lamp with a standard spectrum.

\section{RESULTS}

\section{A. Hall effect}

In GaN:Si,Zn (group A) and GaN:Si samples grown at TUBS, the concentration of free electrons was in the range of $(0.75-1.14) \times 10^{19} \mathrm{~cm}^{-3}$ at room temperature (Table I); i.e., these samples are degenerate. As expected for degenerate semiconductors, the temperature dependence of the electron concentration is negligible (Fig. 1). The Hall mobility in these samples was about $150 \mathrm{~cm}^{2} / \mathrm{Vs}$ at room temperature and slightly increased with decreasing temperature (Fig. 2).

Sample 2015 grown by the HVPE method at TDI contained a thin degenerate layer near the GaN/sapphire interface. This layer shunted the conductivity of the $5 \mu \mathrm{m}$-thick layer, indicated by a rise in the calculated concentration of free electrons with decreasing temperature. ${ }^{7}$ Figures 1 and 2

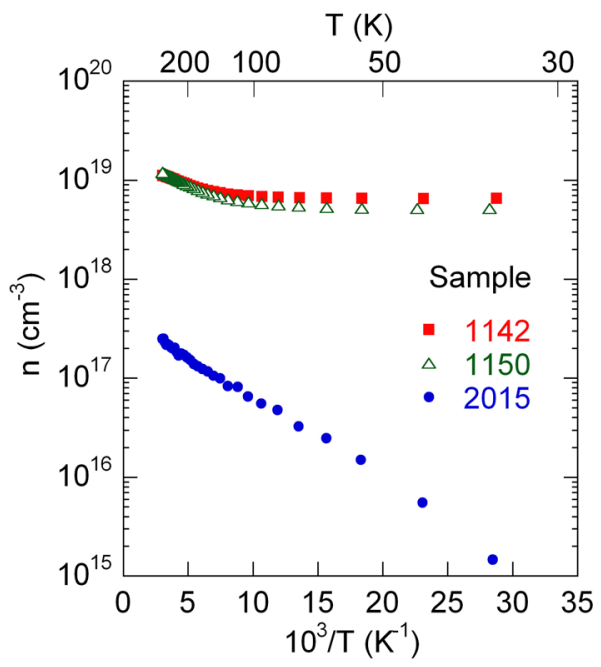

FIG. 1. Temperature dependence of the free-electron concentration in $\mathrm{GaN}: \mathrm{Si}, \mathrm{Zn}$ (sample 1142) and GaN:Si (1150) grown by MOVPE at TUBS and GaN:Si (2015) grown by HVPE at TDI. The data for sample 2015 are calculated by using a two-layer model. ${ }^{7,8}$ show the corrected concentration of free electrons and the electron Hall mobility for the bulk region, excluding the degenerate layer. The description of the two-layer model can be found in Ref. 7, and a more detailed analysis of the Hall effect data for sample 2015 can be found in Ref. 8 .

\section{B. Steady-state photoluminescence}

PL spectra from GaN:Si,Zn samples of group A at 200 and $320 \mathrm{~K}$ are shown in Fig. 3. The PL spectra from these samples were very similar to the spectra obtained from samples of group B. The PL spectra include a strong BL band with a maximum at $2.9 \mathrm{eV}$; a weak peak at higher photon energies, hereafter called the near-band edge (NBE) emission; and a broad yellow luminescence (YL) band with a maximum at about $2.1-2.2 \mathrm{eV}$. The YL band is attributed to a deep acceptor, which is most likely due to a complex defect involving a Ga vacancy. ${ }^{9}$ The BL band is attributed to transitions from the conduction band (or shallow donors at very low temperatures) to the $\mathrm{Zn}_{\mathrm{Ga}}$ acceptor. ${ }^{9,10}$ The shape of this band at different temperatures is well known, 9,10 and this information was used to deconvolute the bands before finding their integrated intensities in this work.

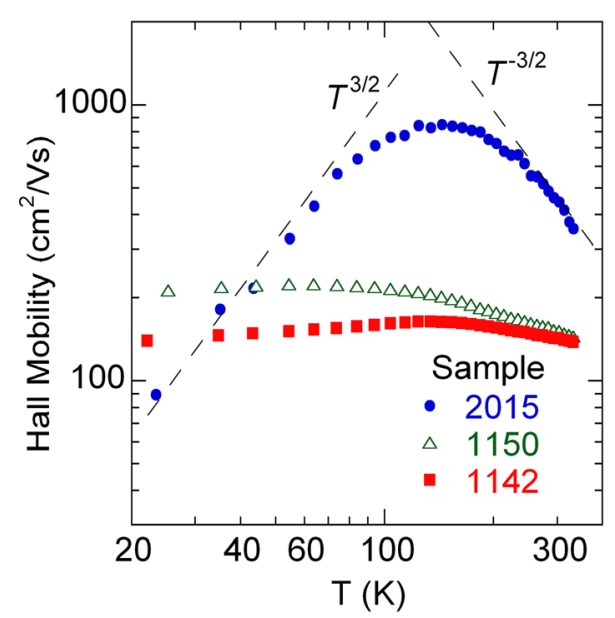

FIG. 2. Temperature dependence of the Hall mobility of electrons in three representative samples. The data for sample 2015 are calculated by using a two-layer model. ${ }^{7,8}$ 

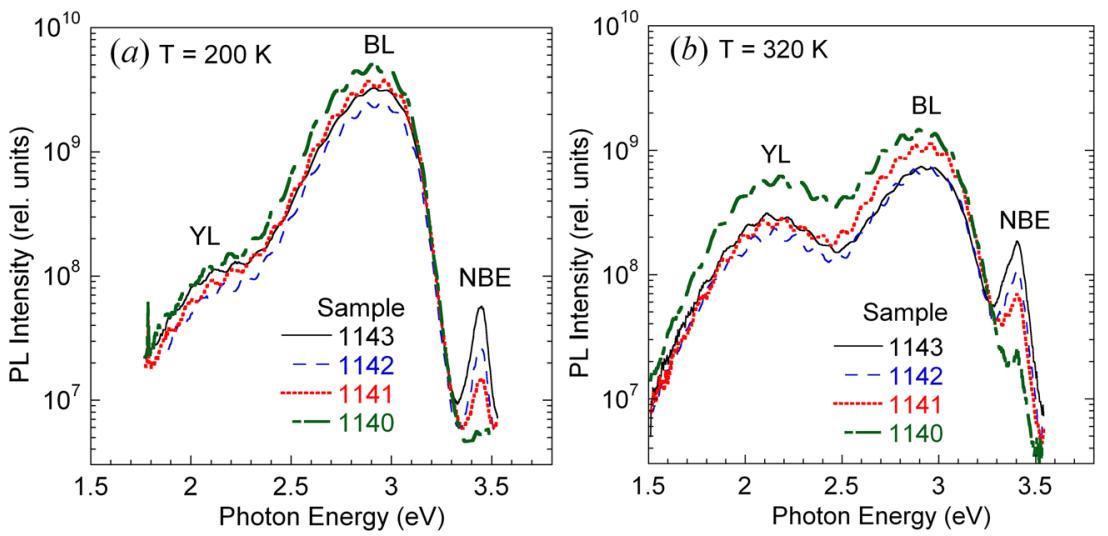

FIG. 3. PL spectra from four GaN:Si,Zn samples of group $\mathrm{A}$ at $200 \mathrm{~K} \quad$ (a) and $320 \mathrm{~K} \quad$ (b). $P_{e x c}=0.0015 \mathrm{~W} / \mathrm{cm}^{2}$.

The excitonic PL spectrum at low temperature is shown in Fig. 4 for sample 1142 at different excitation intensities. Two peaks could be identified in the spectrum. A peak at $3.456 \mathrm{eV}$ is labeled as the acceptor bound exciton (ABE) and is attributed to an exciton bound to the $\mathrm{Zn}_{\mathrm{Ga}}$ acceptor. The source of the peak at $3.484 \mathrm{eV}$ is inconclusive: it might be due to the donor bound exciton (DBE), a free exciton (FE), or a combination of both. The peak at $3.456 \mathrm{eV}$ is seen only at high excitation intensities when the $\mathrm{Zn}_{\mathrm{Ga}}$ acceptors become saturated with holes, while the peak at $3.484 \mathrm{eV}$ dominates at low excitation intensities.

PL spectra at $200 \mathrm{~K}$ for GaN samples doped only with $\mathrm{Si}$ are shown in Fig. 5. In the case of the MOVPE-grown GaN:Si sample, the NBE band was much stronger than that in the GaN:Si,Zn samples. Very small contributions of the BL band can be found near $2.9 \mathrm{eV}$ in sample 1150 (shown with a dashed line in Fig. 5). The signal at $2.9 \mathrm{eV}$ was independent of temperature between 160 and $220 \mathrm{~K}$ and was quenched at higher temperatures with an activation energy approximately equal to that of the BL band in other samples.

The PL spectrum of the HVPE-grown sample (2015) contains a weak and relatively narrow NBE band and a strong BL band. We have established that undoped and

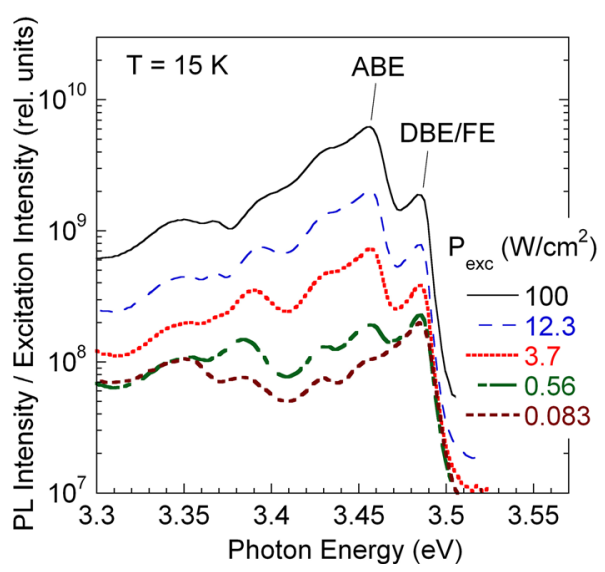

FIG. 4. Excitonic PL spectrum at $T=15 \mathrm{~K}$ and different excitation intensities for sample 1142 . The PL intensity is normalized by dividing each spectrum by the corresponding excitation intensity. ABE is the acceptor $\left(\mathrm{Zn}_{\mathrm{Ga}}\right)$ bound exciton, $\mathrm{DBE}$ is the donor $\left(\mathrm{Si}_{\mathrm{Ga}}\right)$ bound exciton, $\mathrm{FE}$ is free exciton.
Si-doped GaN samples from TDI typically contain residual $\mathrm{Zn}$ (with concentrations on the order of $10^{15} \mathrm{~cm}^{-3}$ ) which is responsible for the strong BL band..$^{9-11}$ At lower photon energies, we can see the red luminescence (RL) band of unknown origin which is also commonly present in undoped and $\mathrm{Si}$-doped GaN samples grown at TDI.

Figure 6 shows the temperature dependence of the integrated PL intensity for the BL band in four GaN:Si,Zn samples, normalized at lower temperatures. The BL band intensity remains nearly constant when the temperature is increased from 15 to $250 \mathrm{~K}$. This band is quenched at $T>$ $250 \mathrm{~K}$ with an activation energy of about $300-340 \mathrm{meV}$. The characteristic temperature of the quenching, $T_{0}$, can be defined as the point where the low-temperature and hightemperature parts of the dependence cross when extrapolated as straight lines in the Arrhenius plot (Fig. 6). This value is unusually high $\left(T_{0}=300 \pm 10 \mathrm{~K}\right)$ in the GaN:Si,Zn samples studied in this work (Table I), as compared to the earlier reported values $\left(T_{0}=200-220 \mathrm{~K}\right) .^{9,12}$ As a comparison, we included in Fig. 6 the data for the Si-doped GaN (sample \#2015) grown at TDI. Clearly, the $T_{0}$ in the GaN:Si,Zn samples is much higher than in samples with low concentrations of $\mathrm{Zn}$ and free electrons.

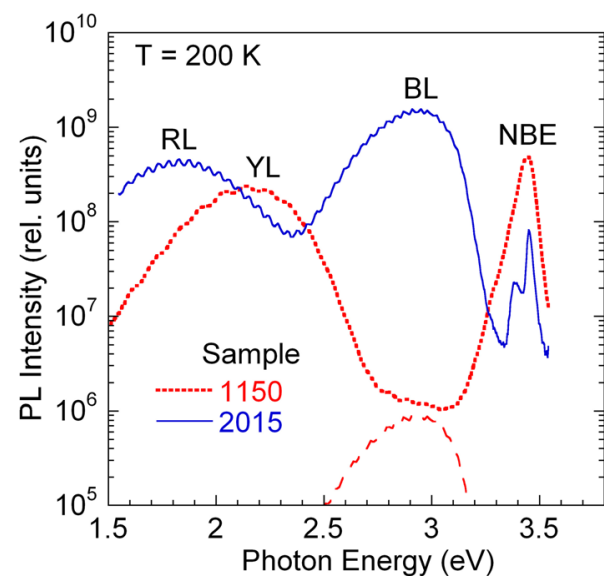

FIG. 5. PL spectra at $200 \mathrm{~K}$ for Si-doped GaN grown by MOVPE at TUBS (sample 1150) and HVPE at TDI (sample 2015). $P_{\text {exc }}=0.0015 \mathrm{~W} / \mathrm{cm}^{2}$. Dashed curve shows the estimated contribution of the BL band to the spectrum of sample 1150 as found from a comparison of the PL spectra at different temperatures. 


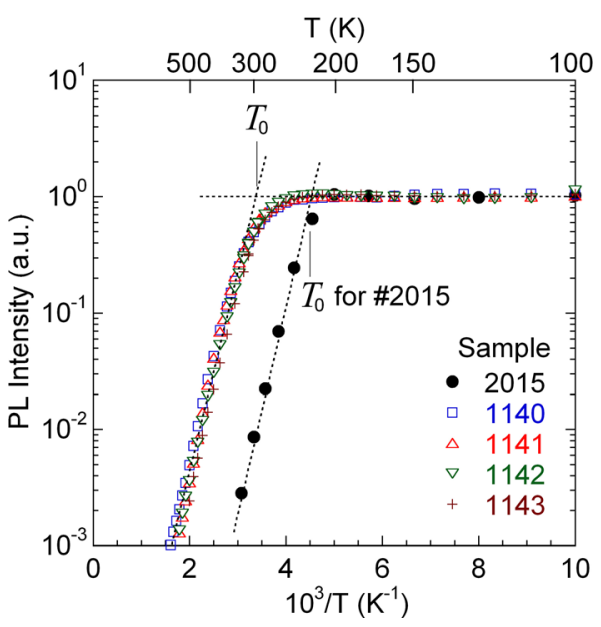

FIG. 6. Temperature dependence of the integrated PL intensity (normalized at temperatures below $100 \mathrm{~K}$ ) for the $\mathrm{BL}$ band in four $\mathrm{GaN}: \mathrm{Si}, \mathrm{Zn}$ samples of group A and GaN:Si sample (\#2015). $P_{\text {exc }}=0.0015 \mathrm{~W} / \mathrm{cm}^{2} . T_{0}=220 \mathrm{~K}$ for sample 2015 , and $T_{0} \approx 300 \mathrm{~K}$ for all other samples.

From 15 to $200 \mathrm{~K}$, the NBE and YL band intensities slowly decrease with increasing temperature. However, at $T=250-350 \mathrm{~K}$, simultaneously with the quenching of the BL band, both the NBE and YL bands exhibit a sharp rise by an order of magnitude and then decrease again at higher temperatures (Fig. 7). This stepwise behavior clearly indicates a very high IQE of the BL band. ${ }^{12}$ In contrast, the intensity of the NBE and YL bands in the Si-doped GaN sample (1150) monotonously decreased with increasing temperature from 100 to $700 \mathrm{~K}$, and no stepwise behavior was observed. The YL band in this sample was quenched at temperatures above $500 \mathrm{~K}$ with an activation energy of $500-550 \mathrm{meV}$, which is very similar to the simulated curve $\eta_{20}(T)$ shown in Fig. 7. Detailed analysis of the YL band behavior in undoped and

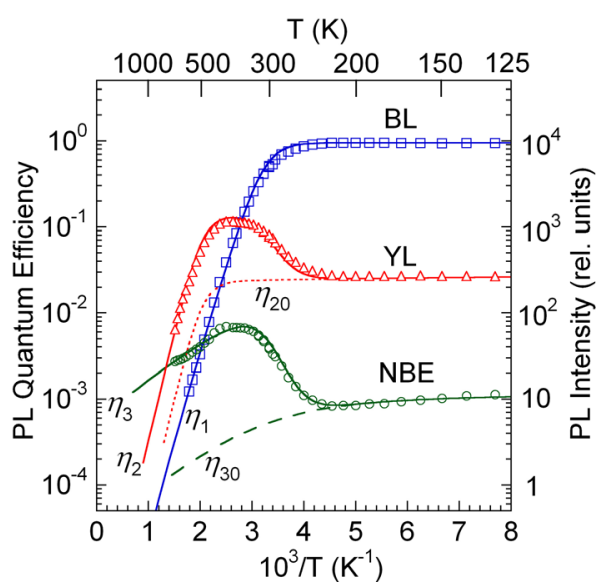

FIG. 7. Temperature dependence of the absolute IQE of PL in GaN:Si,Zn (sample 1141) at $P_{e x c}=0.0015 \mathrm{~W} / \mathrm{cm}^{2}$. Points are experimental data. Solid blue curve shows $\eta_{1}$ calculated by using Eq. (7) with the following parameters: $\eta_{10}=0.91, \quad g=2, \quad C_{p A}=7 \times 10^{-7} \mathrm{~cm}^{3} / \mathrm{s}, \quad \tau_{1}=0.7 \quad \mu \mathrm{s}$, $E_{A 1}=340 \mathrm{meV}$. Dotted red and dashed green lines show $\eta_{20}(T)$ and $\eta_{30}(T)$ calculated by using Eq. (14) with the following parameters: $\eta_{20}(0)=0.027$, $C_{1}=0.3, C_{2}=1.7 \times 10^{5}, E_{1}=15, E_{2}=500 \mathrm{meV}$ (for the YL band); $\eta_{30}(0)=1.2 \times 10^{-3}, C_{1}=1, C_{2}=40, E_{1}=25, E_{2}=100 \mathrm{meV}$ (for the NBE band). $G=2.4 \times 10^{20} \mathrm{~cm}^{-3} \mathrm{~s}^{-1}$ and $\beta=4.5 \times 10^{-17}$ for all the bands. Solid red and green lines show $\eta_{2}(T)$ and $\eta_{3}(T)$ calculated by using Eq. (17) with $\eta_{10}=0.83$ and $\eta_{10}=0.95$, respectively.
Si-doped GaN samples is beyond the scope of this work and will be reported elsewhere.

\section{Time-resolved photoluminescence}

In the studied samples, after pulsed excitation, the BL band intensity decays nearly exponentially. To more accurately find the characteristic lifetime of the BL band, we plotted the product of PL intensity and time as a function of time delay. The PL lifetime was determined from the position of the maximum in this plot. ${ }^{13}$ The PL lifetime is almost constant at $T<T_{0}$ (Fig. 8). However, at temperatures above $T_{0}$, the lifetime decreases with a slope corresponding to the activation energy of the $\mathrm{Zn}_{\mathrm{Ga}}$ acceptor. The temperature dependencies of the PL lifetime and PL intensity were very similar to each other.

The PL lifetime in $n$-type semiconductors is inversely proportional to the concentration of free electrons. ${ }^{13}$ The weak decrease of the PL lifetime with increasing temperature from 100 to $200 \mathrm{~K}$ in sample 2015 can be explained by the corresponding increase in the concentration of free electrons. In the degenerate sample 1142, the PL lifetime was nearly independent of temperature, in the range of 100-250 K, which agrees with the constant concentration of free electrons in this sample.

\section{Dependence of the photoluminescence intensity on excitation intensity}

We have also studied the effect of excitation intensity on the PL intensity for each PL band at different temperatures. The YL band intensity increases linearly at low excitation intensities but begins saturating at $P_{\text {exc }}>10^{-4}-10^{-3} \mathrm{~W} / \mathrm{cm}^{2}$. This saturation can be explained by a number of reasons, including the following: a low concentration of the related defects, the long PL lifetime (greater than $10 \mu \mathrm{s}$ ) and the possible contribution from donor-acceptor pair transitions to this band. In contrast, the BL and NBE band intensities increase linearly with the excitation intensity up to $0.1-1 \mathrm{~W} / \mathrm{cm}^{2}$. Figure 9 shows the ratio of the integrated PL intensity and the excitation intensity as a function of the excitation power density for the NBE and BL bands in four GaN:Si,Zn samples of group A at $200 \mathrm{~K}$. This ratio is proportional to the

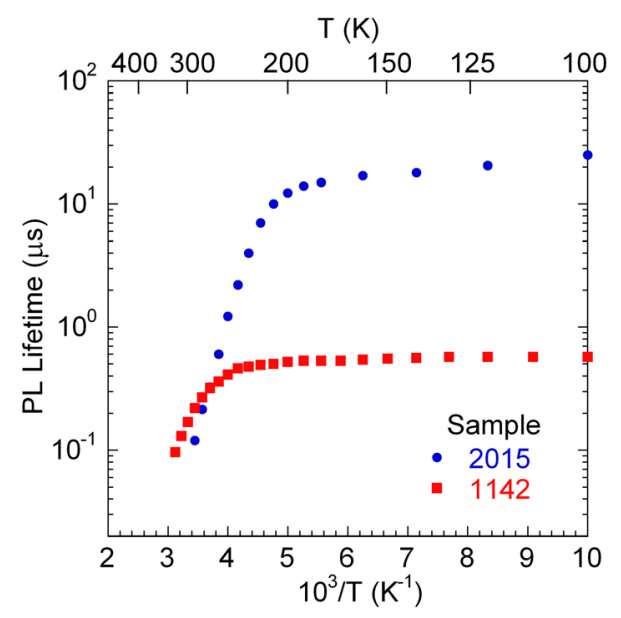

FIG. 8. Temperature dependence of PL lifetime for the BL band in GaN. 


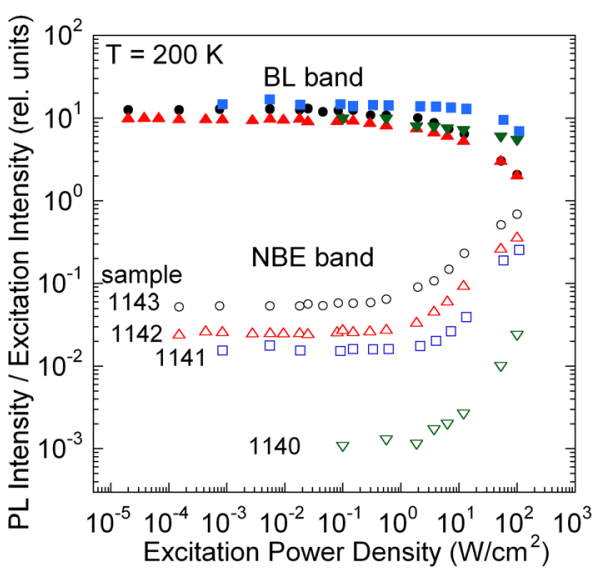

FIG. 9. Integrated PL intensity divided by the excitation intensity as a function of excitation power density for NBE and BL bands in four GaN:Si,Zn samples at $T=200 \mathrm{~K}$. Solid symbols represent the BL band and empty symbols represent the NBE band.

IQE of PL. We can see that the IQE of the BL band starts decreasing above $P_{\text {exc }} \approx 1 \mathrm{~W} / \mathrm{cm}^{2}$. Simultaneously, the IQE of the NBE band increases. As will be shown in Sec. IV C, this behavior is the result of a competition between recombination channels, and it also indicates a very high IQE of the BL band.

\section{MODEL}

\section{A. Rate equations}

Let us consider $n$-type $\mathrm{GaN}$ which contains shallow $\mathrm{Si}_{\mathrm{Ga}}$ donors with concentration $N_{D}, \mathrm{Zn}_{\mathrm{Ga}}$ acceptors responsible for the BL band with concentration $N_{A 1}$, deep acceptors responsible for the YL band with concentration $N_{A 2}$, and one (dominant) type of nonradiative centers, $S$, with concentration $N_{S}$. The variables $N_{D}^{+}, N_{D}^{0}, N_{A 1}^{-}, N_{A 1}^{0}, N_{A 2}^{-}, N_{A 2}^{0}, N_{S}^{-}$, and $N_{S}^{0}$ are the concentrations of these centers in different charge states. For definiteness, we assumed that the nonradiative centers are acceptors in an $n$-type semiconductor.

Under continuous illumination, electron-hole pairs are produced with a generation rate $G$ per unit volume. The steady-state concentrations of free electrons and holes are $n$ and $p$, respectively. We traditionally describe the transition rates as the product of the concentrations of available carriers and available empty sites, multiplied by a constant factor called the capture coefficient. ${ }^{14,15}$ In particular, holes are captured by the $\mathrm{Zn}_{\mathrm{Ga}}$ acceptors, YL-related acceptors, and nonradiative centers at rates $C_{p A 1} N_{A 1}^{-} p, C_{p A 2} N_{A 2}^{-} p$, and $C_{p S} N_{S}^{-} p$, where $C_{p A 1}, C_{p A 2}$, and $C_{p S}$ are the hole-capture coefficients for the $\mathrm{Zn}_{\mathrm{Ga}}$ acceptor, the YL-related acceptor, and the nonradiative center $S$, respectively. In addition, free holes and electrons may recombine directly or form excitons. The rate of these processes will be described as Bnp, where the rate coefficient $B$ includes both the exciton and free electron-hole recombination components. ${ }^{16}$ Holes captured by acceptors, nonradiative defects, or excitons, may return to the valence band as a result of thermal excitation. We will explicitly consider only the thermal emission of holes from the $\mathrm{Zn}_{\mathrm{Ga}}$ acceptors to the valence band at the rate $Q_{A 1},{ }^{10}$ where $Q_{A 1}$ is defined as

$$
Q_{A 1}=C_{p A 1} N_{v} g^{-1} \exp \left(-\frac{E_{A 1}}{k T}\right)
$$

where $E_{A 1}$ is the ionization energy of the $\mathrm{Zn}_{\mathrm{Ga}}$ acceptor, $N_{v}$ is the effective density of states in the valence band, $g$ is the degeneracy factor of the acceptor level (assumed to be equal to 2), $T$ is the temperature, and $k$ is Boltzmann's constant.

The rate equations under steady-state conditions can be constructed with the same approach as in Refs. 10 and 12. The rate of change of the free-hole concentration in the valence band in the steady-state condition is given by

$$
\begin{aligned}
\frac{d p}{d t}= & G-C_{p A 1} N_{A 1}^{-} p-C_{p A 2} N_{A 2}^{-} p-C_{p S} N_{S}^{-} p \\
& -B n p+Q_{A 1} N_{A 1}^{0}=0 .
\end{aligned}
$$

The rate of change of the free electron concentration in the conduction band is given by

$$
\frac{d n}{d t}=G-C_{n A 1} N_{A 1}^{0} n-C_{n A 2} N_{A 2}^{0} n-C_{n S} N_{S}^{0} n-B n p=0,
$$

where $C_{n A 1}, C_{n A 2}$, and $C_{n S}$ are the electron capture coefficients for the $\mathrm{Zn}_{\mathrm{Ga}}$ acceptor, the YL-related acceptor, and nonradiative center $S$, respectively. Capture of electrons by shallow donors $D$, and the thermal emission of electrons from shallow donors to the conduction band are not relevant in the case of a degenerate $n$-type semiconductor. It is also assumed that the concentration of photogenerated electrons in the conduction band, $\delta n$, is much smaller than the concentration of free electrons in the dark, $n_{0}$, so that $n \approx n_{0}$. We can also introduce the characteristic lifetime of luminescence which is related to electron transitions from the conduction band to the $\mathrm{Zn}_{\mathrm{Ga}}$ acceptors as $\tau_{A 1}=\left(C_{n A 1} n\right)^{-1} .{ }^{13}$ Similarly, the characteristic lifetimes of the YL and NBE emission and nonradiative recombination, can be defined as $\tau_{A 2}=\left(C_{n A 2} n\right)^{-1}$, $\tau_{N B E}=(B n)^{-1}$ and $\tau_{S}=\left(C_{n S} n\right)^{-1}$, respectively.

To simplify the notation in Eqs. (2) and (3), we will number the recombination channels via the $\mathrm{Zn}_{\mathrm{Ga}}$ acceptor, YL-related acceptor, exciton (or free electron-hole recombination), and nonradiative center $S$ as $1,2,3$, and 4, respectively. Then, we will let $C_{p i}(i=1,2,3,4)$ represent $C_{p 1} \equiv C_{p A 1}, C_{p 2} \equiv C_{p A 2}, C_{p 3} \equiv B, C_{p 4} \equiv C_{p S} ; \tau_{i}$ represent $\tau_{1} \equiv \tau_{A 1}, \tau_{2} \equiv \tau_{A 2}, \tau_{3} \equiv \tau_{N B E}, \tau_{4} \equiv \tau_{S}$; and $Q_{1} \equiv Q_{p A 1}$. Further, in Eq. (2) we let $N_{i}^{-}$represent $N_{A 1}^{-}, N_{A 2}^{-}, n$, and $N_{S}^{-}$, while in Eq. (3) we let $N_{i}^{0}$ represent $N_{A 1}^{0}, N_{A 2}^{0}, p$, and $N_{S}^{0}$, for the channels with $i=1,2,3$, and 4 , respectively. By using this compact notation for the four recombination channels, Eqs. (2) and (3) can be replaced with the following expressions:

$$
\frac{d p}{d t}=G-\sum_{i=1}^{4} C_{p i} N_{i}^{-} p+Q_{1} N_{1}^{0}=0
$$

and

$$
\frac{d n}{d t}=G-\sum_{i=1}^{4} \frac{N_{i}^{0}}{\tau_{i}}=0 .
$$


The four main recombination channels in GaN:Si,Zn have the absolute IQE $\eta_{i}$. The intensity of the BL band, $I_{1}$, is

$$
I_{1}=\frac{N_{1}^{0}}{\tau_{1}}=C_{p 1} N_{1}^{-} p-Q_{1} N_{1}^{0}
$$

and its IQE is $\eta_{1}=I_{1} / G$. The temperature dependence of the IQE of the BL band, $\eta_{1}(T)$, in $n$-type GaN can be described with the formula ${ }^{10,12}$

$$
\eta_{1}(T)=\frac{\eta_{10}}{1+\left(1-\eta_{10}\right) \tau_{1} Q_{1}},
$$

where $\eta_{10}$ is the IQE of the BL band at low temperatures $(T<200 \mathrm{~K})$ when $Q_{1} \approx 0$. When this dependence is plotted as an Arrhenius plot (logarithm of PL intensity or IQE as a function of inverse temperature), two regions can be distinguished: a region where the IQE of the BL band is independent of temperature at $T<T_{0}$,

$$
\eta_{1}\left(T<T_{0}\right)=\eta_{10}=C_{p 1} N_{1}^{-} p / G,
$$

and a region where the BL band is quenched with an activation energy close to $E_{A 1}$

$$
\eta_{1}\left(T>T_{0}\right) \approx \frac{\eta_{10} g}{\left(1-\eta_{10}\right) \tau_{1} C_{p 1} N_{v}} \exp \left(\frac{E_{A 1}}{k T}\right) .
$$

Note that in Eq. (9) the temperature dependence of $N_{v}$ is weak as compared to the exponential dependence. The characteristic temperature $T_{0}$ can be found from the condition

$$
\left(1-\eta_{10}\right) \tau_{1} Q_{1}=1
$$

and defined as the temperature at which the low-temperature and high-temperature parts in the Arrhenius plot cross when extrapolated as straight lines (Fig. 6).

The IQE of the YL band is

$$
\eta_{2}=C_{p 2} N_{2}^{-} p / G
$$

and the IQE of the NBE emission is

$$
\eta_{3}=B n p / G,
$$

where the coefficient $B$ is a function of temperature.

Finally, the absolute efficiency of the nonradiative recombination is

$$
\eta_{4}=C_{p 4} N_{4}^{-} p / G
$$

The PL intensity integrated over a spectral region of a particular PL band, $I_{i}^{P L}$, is proportional to its absolute IQE, and can be expressed as $I_{i}^{P L}=\beta \eta_{i} G$, where $\beta$ is a constant determined by the total collection efficiency of luminescence (assumed to be independent of temperature and the same for all channels).

\section{B. Dependence of the PL intensity on temperature}

We define $\eta_{i 0}$ and $I_{i 0}^{P L}\left(I_{i 0}^{P L}=\beta \eta_{i 0} G\right)$ as the temperaturedependent absolute IQE and corresponding integrated PL intensity for the $i$ th channel $(i=1,2,3)$ in the case where the $\mathrm{BL}$ band is not quenched and the subsequent redistribution of the minority carriers (holes) between different channels does not occur. However, the quenching of the BL band does cause a redistribution of the holes, which consequently changes the efficiencies of all recombination channels. The temperature-dependent values $\eta_{i}$ and $I_{i}^{P L}$ will include the effect of the BL band quenching.

In practice, the temperature dependence of $I_{i 0}^{P L}$ for $i \neq 1$ can be found by modeling the experimentally found $I_{i}^{P L}(T)$ dependence at temperatures below the quenching of the $\mathrm{BL}$ band with the following expression:

$$
I_{i 0}^{P L}(T)=\frac{I_{i 0}^{P L}(0)}{1+A_{1} e^{-E_{1} / k T}+A_{2} e^{-E_{2} / k T}}
$$

and by extrapolating this dependence to higher temperatures. Here, $I_{i 0}^{P L}(0)=\beta \eta_{i 0}(0) G$ is the integrated PL intensity in the low-temperature limit and $A_{1}, A_{2}, E_{1}$, and $E_{2}$ are fitting parameters that determine the positions and slopes of the lines in the Arrhenius plot. The $I_{i 0}^{P L}(T)$ [or $\eta_{i 0}(T)$ ] dependencies for the YL $(i=2)$ and NBE $(i=3)$ bands are shown in Fig. 7 with dotted and dashed curves, respectively. By using this formal approach, we leave out the explanation of the changes in PL intensities with temperature for the PL bands with $i \neq 1$, which are not directly related to the quenching of the BL band but may be related to dissociation of excitons (for the NBE emission), slow variation in coefficients $C_{p i}$, or any other reasons. Moreover, since the thermal emission of holes to the valence band was not explicitly included in Eq. (4) for channels with $i \neq 1$, we can postulate that the temperature dependence described with Eq. (14) is included in parameters $B$ and $C_{p i}$ in Eqs. (11)-(13).

Since $\sum_{i=1}^{4} \eta_{i}=1$, and each term $\eta_{i}$ with $i>1$ contains the hole concentration $p$ as a factor, we can express $p$ from Eqs. (4) and (6) as

$$
p=\frac{G\left(1-\eta_{1}\right)}{C_{p 2} N_{2}+B n+C_{p 4} N_{4}},
$$

where we replaced $N_{i}^{-}$with $N_{i}$, which is justified for low excitation intensities when defects are not saturated with holes and the relative concentration of holes bound to the YLrelated acceptor and nonradiative center $S$ is negligible. Then, we can substitute $p$ from Eq. (15) into the expressions for the IQE of any channel with $i \neq 1$ [Eqs. (11)-(13) with $N_{i}^{-}=N_{i}$. In the region of substantial thermal quenching of the BL band $(T \approx 250-350 \mathrm{~K})$, the intensities of other PL bands increase, because $\eta_{1}$ in Eq. (15) decreases. If the BL band was not quenched, concentration of holes $p_{0}$ would be described by Eq. (15) with $\eta_{1}=\eta_{10}$; i.e.,

$$
p_{0}=\frac{G\left(1-\eta_{10}\right)}{C_{p 2} N_{2}+B n+C_{p 4} N_{4}},
$$


and the efficiencies of channels with $i=2,3$, and 4 would be, $\eta_{20}=C_{p 2} N_{2} p_{0} / G, \eta_{30}=B n p_{0} / G$, and $\eta_{40}=C_{p 4} N_{4} p_{0} / G$, respectively. Thus, by dividing $\eta_{i}$ by $\eta_{i 0}$ and canceling out the terms that have the same temperature dependence, we can express the relative change of the integrated PL intensity $I_{i}^{P L} / I_{i 0}^{P L}(i=2,3)$ due to the quenching of the BL band as

$$
\frac{I_{i}^{P L}}{I_{i 0}^{P L}}=\frac{\eta_{i}}{\eta_{i 0}}=\frac{1-\eta_{1}}{1-\eta_{10}} .
$$

Note that, according to Eq. (17), the $I_{i}^{P L} / I_{i 0}^{P L}$ ratios and their temperature dependencies are identical for all of the recombination channels with $i>1$. Recalling that $\eta_{1}=\left(I_{1}^{P L} / I_{10}^{P L}\right) \eta_{10}$, we see that $\eta_{10}$ is the only fitting parameter in the fit of the experimentally found $I_{i}^{P L}(T)$ dependencies with Eq. (17) for channels with $i>1$, provided that we have the temperature dependencies of $I_{1}^{P L} / I_{10}^{P L}$ and $I_{i 0}^{P L}(T)$ in relative units.

\section{Dependence of PL intensity on excitation intensity}

Remarkably, Eq. (17) can be used not only in the case of the thermal quenching of the BL band but also in the case of its "quenching" by high excitation intensity. Indeed, the IQE of the defect-related PL band decreases with increasing excitation intensity above some critical value $G_{0}$, due to saturation of the defect with photogenerated holes. ${ }^{9}$ The dependence of $\eta_{1}$ on $G$ can be described with a simple model which assumes that within an active layer of thickness $\alpha^{-1}$ the generation rate $G$ is constant, where $\alpha$ is the absorption coefficient. In application to the BL band in GaN:Si,Zn, it can be shown that the $\eta_{1}(G)$ dependence is ${ }^{9}$

$$
\eta_{1}=\frac{1}{2}\left(1+\frac{N_{1}}{G \tau_{1} \eta_{10}}\right)-\sqrt{\frac{1}{4}\left(1+\frac{N_{1}}{G \tau_{1} \eta_{10}}\right)^{2}-\frac{N_{1}}{G \tau_{1}}},
$$

where $\eta_{10}$ is the IQE of the BL band in the limit of low excitation intensities when the BL band intensity increases linearly with the excitation intensity. Remarkably, when $\eta_{10}, G$, and $\tau_{1}$ are known, the concentration of the $\mathrm{Zn}_{\mathrm{Ga}}$ acceptors $\left(N_{1}\right)$ can be estimated from the fit of the $\eta_{1}(G)$ dependence with Eq. (18).

However, the above approximation introduces some error because the complete saturation of the PL intensity at $G \gg G_{0}=N_{1} /\left(\tau_{1} \eta_{10}\right)$ predicted by Eq. (18) is never observed in the experiment. This is because the excitation intensity decreases exponentially inside the semiconductor. ${ }^{17}$ This phenomenon can be accounted for by replacing $G$ with $G(x)$, where $x$ is the distance from the semiconductor surface into the bulk, and by integrating Eq. (18) over the entire thickness of a semiconductor layer. ${ }^{17}$ In this method, we plot the IQE of the PL as a function of the excitation intensity $P_{0}=P_{\text {exc }} / h \nu_{\text {exc }}$, where $h \nu_{\text {exc }}$ is the photon energy of the excitation light, and is expressed by the number of photons per unit time, per unit area. An example of the fit of the $\eta_{1}\left(P_{0}\right)$ dependence with the numerical integration of Eq. (18) [in which $G(x)=\alpha P_{0} e^{-\alpha x}$ ] is shown in Fig. 10 .

The saturation of the BL band intensity (or decrease of its IQE) with increasing excitation intensity can be inter-

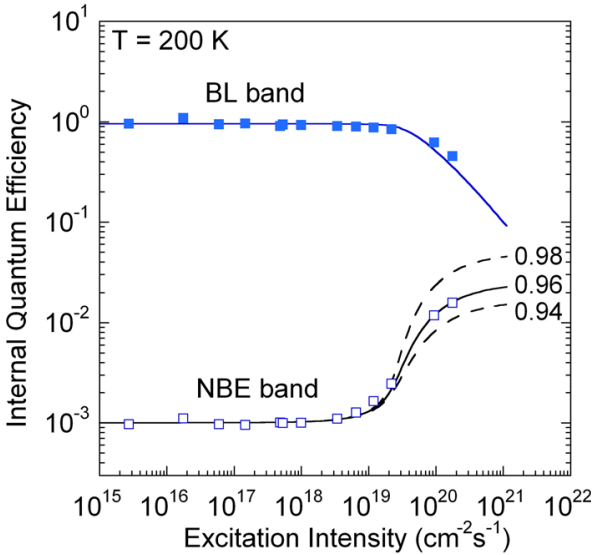

FIG. 10. Dependence of the IQE of PL on excitation intensity for the NBE and BL bands in GaN:Si,Zn (sample \# 1141) at $T=200 \mathrm{~K}$. Fit of the BL band is obtained by integrating Eq. (18) over the sample thickness with the following parameters: $\tau_{1}=0.7 \mu \mathrm{s}, \eta_{10}=0.96$, and $N_{1}=1.5 \times 10^{18} \mathrm{~cm}^{-3}$. Solid curve for the NBE band shows $\eta_{3}(G)$ calculated by using Eq. (17) with $\eta_{30}=10^{-3}$ and $\eta_{10}=0.96$. Dashed curves show the fit with $\eta_{10}=0.98$ and 0.94 , as indicated in the figure.

preted as the quenching of the BL-related recombination channel. This effect is very similar to the thermal quenching of the BL band. Thus, it should result in an equal rise in the recombination rate for all other channels. The latter is described with Eq. (17) which is the same for the quenching of the BL band by temperature and excitation intensity, because it does not distinguish between different sources that reduce $\eta_{1}$. From the fit of the experimental $\eta_{i}\left(P_{0}\right)$ dependence with Eq. (17) we can find the IQE of the BL band in the limit of the low excitation intensity $\left(\eta_{10}\right)$. This method is especially good when $\eta_{i 0}(i \neq 1)$ is independent of the excitation intensity for a wide range, which is expected for the NBE emission in a degenerate $n$-type semiconductor.

\section{DISCUSSION}

\section{A. Determination of the absolute IQE of photoluminescence}

We can determine the absolute IQE of different PL bands, as well as the efficiency of nonradiative recombination, from four independent methods: (1) from the position of the characteristic temperature, $T_{0}$, in the $I_{1}^{P L}\left(T^{-1}\right)$ dependence; (2) from the fit of the temperature dependence of the YL band intensity; (3) from the fit of the temperature dependence of the NBE band intensity; and (4) from the fit of the dependence of the NBE band intensity on excitation intensity. The results are summarized in Table II.

\section{Characteristic temperature of the quenching of the $B L$ band}

In the GaN:Si,Zn samples used in this study, the quenching of the $\mathrm{BL}$ band begins at much higher temperatures $\left(T_{0}=300 \mathrm{~K}\right)$ than it is commonly observed $(200-220 \mathrm{~K})^{9,}{ }^{9,13}$ see Fig. 6. This can be partially explained by a short PL lifetime (due to a high concentration of free electrons). However, the main reason for the significant change in $T_{0}$ is the exceptionally high IQE of the BL band. In the fitting of the 
TABLE II. Absolute IQE of PL at $200 \mathrm{~K}$.

\begin{tabular}{|c|c|c|c|c|c|c|c|}
\hline \multirow[b]{2}{*}{ Sample } & \multirow[b]{2}{*}{ DeZn flux } & \multicolumn{5}{|c|}{$\eta_{10}$} & \multirow{2}{*}{$\frac{\eta_{30}}{\text { From } \frac{I_{10}^{P L}}{I_{20}^{P L}}}$} \\
\hline & & From $I_{1}^{P L}(T)$ & From $I_{2}^{P L}(T)$ & From $I_{3}^{P L}(T)$ & From $I_{3}^{P L}\left(P_{0}\right)$ & Weighted average and error & \\
\hline 1143 & 4 & 0.91 & 0.88 & 0.92 & 0.93 & $0.92_{-0.04}^{+0.01}$ & 0.006 \\
\hline 1142 & 10 & 0.94 & 0.83 & 0.92 & 0.95 & $0.94_{-0.03}^{+0.01}$ & 0.0024 \\
\hline 1141 & 33 & 0.91 & 0.83 & 0.95 & 0.96 & $0.95_{-0.03}^{+0.01}$ & 0.001 \\
\hline 1140 & 66 & 0.99 & 0.88 & 0.88 & 0.97 & $0.96_{-0.04}^{+0.01}$ & 0.00013 \\
\hline 1010 & 4 & 0.80 & 0.65 & 0.80 & 0.85 & $0.81_{-0.05}^{+0.02}$ & 0.0042 \\
\hline 1009 & 10 & 0.89 & 0.85 & 0.85 & 0.87 & $0.86_{-0.05}^{+0.02}$ & 0.002 \\
\hline 1008 & 33 & 0.96 & 0.93 & 0.92 & 0.9 & $0.91_{-0.04}^{+0.01}$ & 0.0009 \\
\hline 997 & 66 & 0.94 & 0.91 & 0.94 & 0.93 & $0.93_{-0.05}^{+0.01}$ & 0.00013 \\
\hline 2015 & - & $<0.2$ & $<0.5$ & $<0.5$ & - & $0.3_{-0.2}^{+0.1}$ & \\
\hline
\end{tabular}

BL intensity with Eq. (7), the fitting parameters were $E_{A 1}$ (determines the slope of the high-temperature part of the Arrhenius plot) and $\eta_{10}$ (determines the position of $T_{0}$ ). The value of $C_{p 1}$ for the BL band in undoped $\mathrm{GaN}$ has been estimated as $(9 \pm 3) \times 10^{-7} \mathrm{~cm}^{3} / \mathrm{s}$ in Ref. 12 from analysis of the temperature dependencies of the BL band intensity in four samples grown by the MOVPE method. Since that time, we have analyzed the Zn-related BL band in many other GaN samples, including undoped, Si-doped and $\mathrm{Zn}$-doped GaN grown by the MOVPE and HVPE methods. Based on these extensive data, we will slightly revise the value of $C_{p 1}$, and instead use $C_{p 1}=7 \times 10^{-7} \mathrm{~cm}^{3} / \mathrm{s}$ in this work. The values of $\eta_{10}$ obtained with this method $\left[\eta_{10}\right.$ from $\left.I_{1}^{P L}(T)\right]$ are listed in the third column of Table II. When $\eta_{10}$ is of the order of 0.1 or smaller, only the upper bound for $\eta_{10}$ can be estimated $\left(\eta_{10}<0.2\right.$ for sample 2015) because the $I_{1}^{P L}(T)$ dependencies with $\eta_{10}=0.2$ and $\eta_{10}=0$ cannot be distinguished reliably while accounting for reasonable errors in the fixed and fitting parameters.

\section{Enhancement of the $Y L$ band due to the quenching of the $B L$ band}

The intensity of the YL band in GaN:Si, $\mathrm{Zn}$ remained nearly unchanged with increasing temperature from 15 to 250 K. Remarkably, close to $T=T_{0}$, a stepwise rise in the YL band intensity is observed simultaneously with the quenching of the BL band (Fig. 7). At higher temperatures $(T>500 \mathrm{~K})$, the YL band was quenched with the activation energy of $500-600 \mathrm{meV}$ in almost all of the samples. The quenching could be explained by the thermal emission of holes from the YL-related acceptor to the valence band. It is beyond the scope of this work to discuss the details of this quenching and to identify the YL-related defect. What is important is the similar stepwise increase of the YL and NBE emission intensities by about an order of magnitude at temperatures close to $T=T_{0}$, which unambiguously indicates the very high absolute IQE of the BL band. From the fit of the experimental dependence of the YL band intensity with Eq. (17) (shown in Fig. 7 with a red solid curve labeled $\eta_{2}$ ), we find $\eta_{10}=0.83$ for the absolute IQE of the BL band in sample 1141 . The parameter $\eta_{10}$ for other samples was estimated with the same procedure, and the values are listed in column 4 of Table II. There is no YL band in the PL spectrum of sample 2015. However, the RL band showed a small increase (by up to a factor of two) in the region where the BL band was quenched, which allows us to estimate that $\eta_{10}<0.5$ in this sample.

\section{Enhancement of the NBE emission due to the quenching of the $B L$ band}

The integrated intensity of the NBE emission decreases with increasing temperature, which could be attributed to the dissociation of excitons. However, close to $T=T_{0}$, a stepwise rise in the NBE emission intensity is observed simultaneously with the quenching of the BL band (Fig. 7). The only plausible explanation for this rise in intensity is the thermal release of holes from the $\mathrm{Zn}_{\mathrm{Ga}}$ acceptor and their subsequent redistribution between all the other recombination channels. From the value of the step in the NBE emission (about an order of magnitude) we can quickly estimate $^{10}$ that the absolute IQE of the BL band is about $90 \%$. A more accurate value can be obtained by fitting the temperature dependence of the NBE emission intensity with Eq. (17). The temperature dependence of $I_{30}^{P L}$ in this equation is simulated with Eq. (14) in which two activation energies correspond to dissociation of free excitons at $T<T_{0}$ $\left(E_{1}=25 \mathrm{meV}\right)$ and unknown process involving apparent thermal quenching with $E_{1}=100 \mathrm{meV}$ at higher temperatures. The latter is clearly visible as a new slope of the NBE emission intensity decrease at $T>>T_{0}$. The best fit of the $I_{3}^{P L}(T)$ dependence (right scale) with Eq. (17) for the NBE band is shown in Fig. 7 with a green solid curve labeled as $\eta_{3}$. From this fit, we find $\eta_{10}=0.95$ for the absolute IQE of the BL band in this sample. The parameter $\eta_{10}$ for other samples was estimated using the same procedure, and the values are listed in column 5 of Table II. For sample 2015, the $I_{3}^{P L}(T)$ dependence increased by at most a factor of two from the $I_{30}^{P L}(T)$ dependence in the region of the quenching of the BL band, indicating that $\eta_{10}<0.5$.

The estimate of $\eta_{10}$ from the temperature dependence of the NBE band is more accurate than the one obtained from the $I_{2}^{P L}(T)$ dependence. The latter is less accurate because the quenching of the YL band with large activation energy starts at temperatures close to $T_{0}$, which may reduce the step by a value that is difficult to determine without 
additional information about the YL band. For this reason, we assigned larger error bars for $\eta_{10}$ determined from data for the YL band.

\section{Enhancement of the NBE emission due to the saturation of the $B L$ band at high excitation intensity}

At low excitation intensity $\left(P_{e x c}<1 \mathrm{~W} / \mathrm{cm}^{2}\right)$, the IQE of the NBE emission is constant in samples with a high concentration of free electrons (Fig. 9); i.e., the NBE emission intensity increases linearly with the excitation intensity. This agrees with our theoretical prediction for the case when $n \approx n_{0}$ [see Eqs. (12) and (15)]. The IQE of the BL band is also constant at $P_{e x c}<1 \mathrm{~W} / \mathrm{cm}^{2}$. At higher excitation densities, the BL band intensity starts saturating because of the limited number of the $\mathrm{Zn}_{\mathrm{Ga}}$ acceptors. Remarkably, the IQE of the NBE emission increases, simultaneously with the decrease in the IQE of the BL band. The rise in the IQE of the NBE emission is expected to be followed by a constant IQE at higher excitation intensities; yet, they could not be achieved in our experiment. The $I_{3}^{P L}\left(P_{e x c}\right)$ dependence for the NBE band can be fit with Eq. (17) where the $\eta_{1}\left(P_{0}\right)$ is found from the fit of the dependence of the BL band intensity on excitation intensity with the method described in Sec. IV C. The fit with Eq. (17) allows us to find $\eta_{10}$ (see Fig. 10). When $\eta_{10}$ is close to unity, the accuracy of this method is high. The values of $\eta_{10}$ found with this method are listed in the sixth column of Table II. This method has the advantage that $I_{i 0}^{P L}$ for the NBE emission is linearly proportional to $G$ in the entire range of the excitation intensities, due to the high concentration of free electrons in degenerate $n$-type GaN samples. We were not able to obtain useful information with this method in application to sample 2015, because the concentration of free electrons was relatively low, and the $I_{3}^{P L}\left(P_{e x c}\right)$ dependence was slightly superlinear in the entire range of $P_{e x c}$ so that a small step in this dependence might remain unnoticed.

\section{Error analysis}

We have determined the absolute IQE of the BL band by using four independent methods. The results are summarized in Table II and Fig. 11. We can see that the IQE is higher in group A (uncapped layers) and it increases with increasing concentration of $\mathrm{Zn}$. The value of $96 \%$ is the highest absolute IQE of PL reported for GaN in the limit of low excitation intensities. Very close values of $\eta_{10}$ obtained from different methods provide confidence in the validity of these results.

The error in $\eta_{10}$ has been estimated in each of the four approaches for each sample. The weighted average and its error (column 7 in Table II) were calculated following recommendations given in Ref. 18. The error is not symmetrical about the mean value because the accuracy in all the methods improves when $\eta_{10}$ approaches unity. As an example, the error analysis for the sample with the highest IQE of PL (sample 1140) is given below.

From the fit of the temperature dependence of $I_{1}^{P L}$ with Eq. (7) with fixed parameters $\tau_{1}=1.48 \mu$ s and $C_{p A 1}=7 \times 10^{-7} \mathrm{~cm}^{3} / \mathrm{s}$, we find two fitting parameters: $\eta_{10}=0.992$ and $E_{A 1}=290 \mathrm{meV}$. They determine the posi-

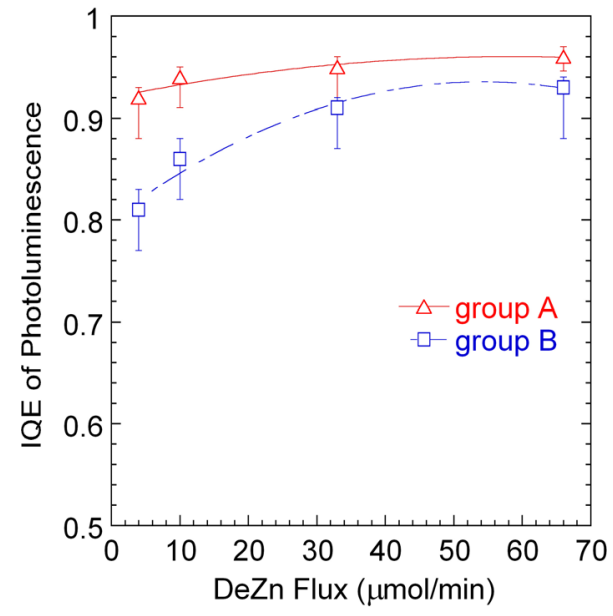

FIG. 11. Absolute IQE of the BL $\left(\eta_{10}\right)$ in GaN:Si,Zn samples of groups A and B. $T=200 \mathrm{~K}$ and $P_{e x c}=0.0015 \mathrm{~W} / \mathrm{cm}^{2}$. The lines are added to guide the eye.

tion and slope, respectively, of the high-temperature part of the dependence. Parameters $C_{p A 1}$ and $\tau_{1}$ in Eq. (7) were estimated from independent experiments as $7 \times 10^{-7} \mathrm{~cm}^{3} / \mathrm{s}$, and $1.48 \mu$ s (see Secs. IIIC and VA1, and Table I). To find uncertainty in $\eta_{10}$, we varied $E_{A 1}$ from 240 to $340 \mathrm{meV}, C_{p A 1}$ - from $3 \times 10^{-7}$ to $1.5 \times 10^{-6} \mathrm{~cm}^{3} / \mathrm{s}$, and $\tau_{1}$ from 1.28 to $1.68 \mu \mathrm{s}$. By varying these three parameters in favorable and unfavorable directions to obtain a higher $\eta_{10}$, we have found $\eta_{10}=0.999$ and $\eta_{10}=0.89$, respectively, i.e., $\eta_{10}=0.992_{-0.10}^{+0.007}$. In similar way, from analysis of the $I_{2}^{P L}(T)$, $I_{3}^{P L}(T)$, and $I_{3}^{P L}\left(P_{0}\right)$ dependencies, we have determined $\eta_{10}=0.88_{-0.20}^{+0.07}, \eta_{10}=0.88_{-0.10}^{+0.05}$, and $\eta_{10}=0.97_{-0.03}^{+0.01}$, respectively, for sample 1140. Finally, the weighted average and propagated uncertainties are found ${ }^{18}$ as $\eta_{10}=0.96_{-0.04}^{+0.01}$ for this sample (Table II). Note that the size of the error bars decreases as the IQE approaches unity.

\section{B. Determination of the concentration of defects and free electrons from photoluminescence}

\section{Determination of the electron concentration from photoluminescence}

Concentration of free electrons can be determined from the following expression: ${ }^{12,13}$

$$
n=\left(C_{n 1} \tau_{1}\right)^{-1} .
$$

Here, the PL lifetime $\tau_{1}$ can be found from the time-resolved PL measurements. The electron-capture coefficient for the $\mathrm{Zn}_{\mathrm{Ga}}$ acceptor, $C_{n 1}$, is a characteristic of the acceptor, and therefore it should not be sample-dependent. Previously, by using Eq. (19), we have determined that $C_{n 1}=4 \times$ $10^{-13} \mathrm{~cm}^{3} / \mathrm{s}$ as the average value for the BL band in four undoped GaN samples grown by MOVPE. ${ }^{9,12,13}$ The new experimental data presented in this work allow us to revise this value. By taking the electron concentration for the GaN:$\mathrm{Si}, \mathrm{Zn}$ samples of group A from the Hall effect data and the lifetime of the BL band from the time-resolved PL data, we find that $C_{n 1}=(1.5 \pm 0.2) \times 10^{-13} \mathrm{~cm}^{3} / \mathrm{s}$. This value is expected to be more accurate than the previously found value 
of $C_{n 1}=4 \times 10^{-13} \mathrm{~cm}^{3} / \mathrm{s}$ for the following reasons. The concentration of electrons in the top $0.2 \mu$ m-thick layer (where most of the PL originates) may be different from the concentration in the remaining part of the 2-5 $\mu$ m-thick "bulk" GaN layer; or it may be shunted by a more conductive layer in nominally undoped GaN samples. However, in the GaN:Si,Zn samples studied in this work, the $0.2 \mu \mathrm{m}$ thick layer was isolated from the undoped $\mathrm{GaN}$ layer by an insulating $\mathrm{AlGaN}$ barrier. Moreover, the high concentration of electrons in the degenerate $\mathrm{GaN}: \mathrm{Si}, \mathrm{Zn}$ layers reduces the roles of the surface, interfaces, and contacts in the determination of the electron concentration by the Hall effect measurements. Furthermore, from the time-resolved PL data for degenerate GaN:Si,Zn samples at $200 \mathrm{~K}$, the decay of the BL intensity, after a laser pulse, was close to an exponential dependence: closer than that in undoped $\mathrm{GaN}$ samples. This better justifies the use of Eq. (19) for degenerate material, because this expression is derived for the recombination of free electrons and bound holes, and may be invalid when the donor-acceptor pair recombination (characterized with a nonexponential decay of PL) is significant.

By using the new value of $C_{n 1}=(1.5 \pm 0.2)$ $\times 10^{-13} \mathrm{~cm}^{3} / \mathrm{s}$ for the $\mathrm{Zn}_{\mathrm{Ga}}$ acceptor and the values of the $\mathrm{BL}$ lifetime at $200 \mathrm{~K}$ determined from the time-resolved PL measurements, we have estimated the concentration of free electrons in $\mathrm{GaN}: \mathrm{Si}, \mathrm{Zn}$ samples of groups A and B (Table I). In these samples, $n \approx 10^{19} \mathrm{~cm}^{-3}$, and the variation in $n$, with increasing temperature from 200 to $300 \mathrm{~K}$, is negligible.

The concentration of free electrons in Si-doped $\mathrm{GaN}$ (sample 2015) has been determined with this method as 4.5 $\times 10^{17} \mathrm{~cm}^{-3}$ at $200 \mathrm{~K}$ and extrapolated to be $6.5 \times 10^{17} \mathrm{~cm}^{-3}$ at room temperature. This value is close to the value obtained from analysis of the temperature-dependent Hall effect data by using a two-layer model $\left(3 \times 10^{17} \mathrm{~cm}^{-3}\right){ }^{8}$

It may be informative to determine some other characteristics of the $\mathrm{GaN}: \mathrm{Si}, \mathrm{Zn}$ samples in the conditions of the steady-state PL. We can estimate the concentration of free holes from Eq. (4) with $Q_{1}=0$ for temperatures below $250 \mathrm{~K}$. It is roughly $10^{6} \mathrm{~cm}^{-3}$ for the lowest excitation density $\left(10^{-5} \mathrm{~W} / \mathrm{cm}^{2}\right)$ and $\sim 10^{13} \mathrm{~cm}^{-3}$ for the highest excitation density used in our experiments $\left(100 \mathrm{~W} / \mathrm{cm}^{2}\right)$. The lifetime of these holes in the valence band at temperatures below $250 \mathrm{~K}$ can be estimated from the following expression:

$$
\tau_{p}=\frac{1}{\sum_{i=1}^{4} C_{p i} N_{i}}=\frac{\eta_{10}}{C_{p 1} N_{1}}
$$

which is on the order of $10^{-13} \mathrm{~s}$. Such short lifetimes of free holes are due to their fast capture by the $\mathrm{Zn}_{\mathrm{Ga}}$ acceptors.

\section{Determination of the concentrations of the $\mathrm{Zn}_{G a}$ and $\mathrm{Si}_{\mathrm{Ga}}$ centers from photoluminescence}

We determined the concentration of the $\mathrm{Zn}_{\mathrm{Ga}}$ centers in GaN:Si,Zn and GaN:Si samples from the PL data by using two methods. In method 1 , the value of $N_{1}$ was determined from the dependence of the BL band intensity on excitation intensity with the approach described in Sec. IV C. The IQE of the BL band was plotted as a function of the excitation intensity $P_{0}$ (Fig. 10). The $\eta_{1}\left(P_{0}\right)$ dependence was fit with numerical integration of Eq. (18), in which $G(x)=\alpha P_{0} e^{-\alpha x}$. With known parameters $\eta_{10}$ and $\tau_{1}$, the only fitting parameter is the concentration of the $\mathrm{Zn}_{\mathrm{Ga}}$ centers, $N_{1}$. The values found with this method are listed in column 6 of Table I and are shown in Fig. 12 for the samples of groups A and B.

In method 2, the value of $N_{1}$ is determined from the comparison of the IQE of the BL and NBE bands at $200 \mathrm{~K}$. At low excitation intensity and at temperatures below the quenching of the BL band, $N_{1}^{-}=N_{1}, \eta_{1}=\eta_{10}$, and $\eta_{3}=\eta_{30}$. Then from Eqs. (8) and (12) we find

$$
N_{1}=\frac{\eta_{10}}{\eta_{30}} \frac{B n}{C_{p 1}} .
$$

The values of $\eta_{10}$ were determined as the weighted average from the four methods described in Sec. V A (Table II). The values of $\eta_{30}$ were estimated from the ratios of the integrated PL intensities for the NBE and BL bands multiplied by $\eta_{10}$, where both $\eta_{10}$ and $\eta_{30}$ were found at $200 \mathrm{~K}$ and at the low excitation intensity limit. The values of $n$ were taken from the time-resolved PL data (Table I). Finally, we assumed that $B=10^{-10} \mathrm{~cm}^{3} / \mathrm{s}$ in GaN at $200 \mathrm{~K}$. The value of $B$ for bandto-band transitions in $\mathrm{GaN}$ is predicted to have the value between $2 \times 10^{-11}$ and $4.5 \times 10^{-10} \mathrm{~cm}^{3} / \mathrm{s}$ for $\mathrm{GaN}$ at $300 \mathrm{~K} .{ }^{16,19-21}$ With decreasing temperature from 300 to $200 \mathrm{~K}, B$ is expected to increase by a factor of two. ${ }^{16,19-21}$ Thus, the value of $B=10^{-10} \mathrm{~cm}^{3} / \mathrm{s}$ at $200 \mathrm{~K}$ is a reasonable value for our rough estimates.

Using the above-described methods, we estimated the concentrations of the $\mathrm{Zn}_{\mathrm{Ga}}$ centers in $\mathrm{GaN}: \mathrm{Si}, \mathrm{Zn}$ samples from groups $\mathrm{A}$ and $\mathrm{B}$, and plotted them as a function of the Zn flow rate during growth (Fig. 12). Although we anticipate an error up to a factor of three for the absolute values of $N_{1}$ determined by each of the above methods, the relative values of the concentration of the $\mathrm{Zn}_{\mathrm{Ga}}$ centers in different samples have much better accuracy. We see that the

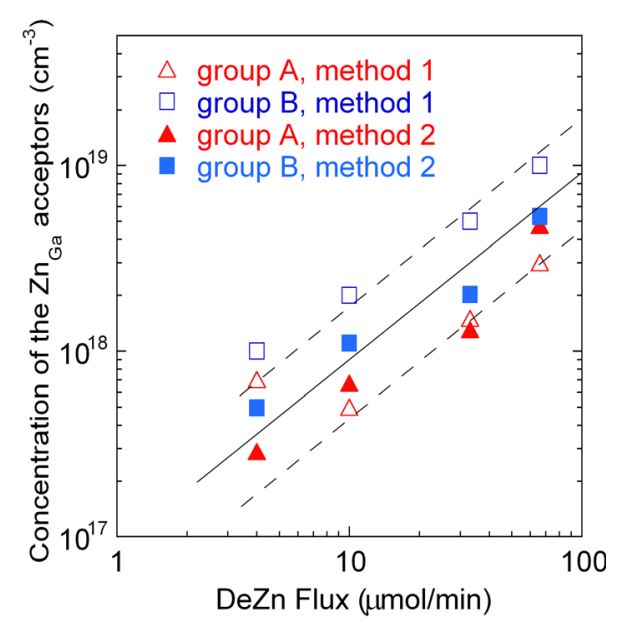

FIG. 12. Concentration of the $\mathrm{Zn}_{\mathrm{Ga}}$ acceptors in $\mathrm{GaN}: \mathrm{Si}, \mathrm{Zn}$ samples of series $\mathrm{A}$ and $\mathrm{B}$ calculated by two methods: from the $\eta_{1}\left(P_{0}\right)$ dependence (method 1) and from comparison of the IQE of the BL and NBE bands (method 2). $T=200 \mathrm{~K}, C_{p 1}=7 \times 10^{-7} \mathrm{~cm}^{3} / \mathrm{s}, B=10^{-10} \mathrm{~cm}^{3} / \mathrm{s}$, The values of $n, \eta_{10}, \eta_{30}$, and $\tau_{1}$ are taken from Tables I and II. Solid and dashed lines show linear dependencies. 
concentration of the $\mathrm{Zn}_{\mathrm{Ga}}$ acceptors increases nearly linearly with DeZn flux and reaches the maximum value of $\sim 10^{19} \mathrm{~cm}^{-3}$ (Fig. 12).

Remarkably, the above-described methods allow us to estimate the concentration of the $\mathrm{Zn}_{\mathrm{Ga}}$ centers when $\mathrm{Zn}$ is present in minute amounts, i.e., when its concentration cannot be determined by other methods. So, we obtained $N_{1}=3 \times 10^{15} \mathrm{~cm}^{-3}$ from method 1 for sample 2015, and an even smaller concentration of $\mathrm{Zn}$ is found in sample 1150 $\left(N_{1}=2 \times 10^{13} \mathrm{~cm}^{-3}\right)$ with method 2 . Note that method 1 cannot be applied to sample 1150 because the BL band could not be reliably resolved at different excitation intensities needed to plot the $I_{1}^{P L}\left(P_{0}\right)$ dependence. On the other hand, method 2 appears to be accurate only for samples with a high concentration of free electrons, when $n \approx n_{0}$. For these samples, band-to-band recombination may dominate over the exciton recombination, at least at 200-300 K, and the surface effects (responsible for the near-surface depletion region) are negligible due to a very small depletion region width.

To find the concentration of the $\mathrm{Si}_{\mathrm{Ga}}$ centers, we need to add $N_{1}$ to $n: N_{S i}=N_{1}+n$. For all the samples of groups A and $\mathrm{B}$, the concentration of the $\mathrm{Si}_{\mathrm{Ga}}$ centers is about $10^{19} \mathrm{~cm}^{-3}$, which is the same as in the sample grown in similar conditions but without $\mathrm{Zn}$ doping (sample 1150). Such a high concentration of shallow donors agrees with the large width of the NBE emission band at $200 \mathrm{~K}$ [compare the shape of the NBE band for samples 2015 and 1150 in Fig. 5 and also for GaN:Si,Zn samples of group A in Fig. 3(a)]. Note that the total concentrations of $\mathrm{Si}$ and $\mathrm{Zn}$ impurities in the $\mathrm{GaN}: \mathrm{Si}, \mathrm{Zn}$ layer may be higher than the concentrations of the $\mathrm{Si}_{\mathrm{Ga}}$ and $\mathrm{Zn}_{\mathrm{Ga}}$ centers, respectively, because not all impurities occupy substitution positions.

\section{Other defects in $\mathrm{GaN}: \mathrm{Si}, \mathrm{Zn}$}

We assume that the YL band in the GaN samples heavily doped with $\mathrm{Si}$ is associated with the Si-related defects, and not with $\mathrm{C}$ or the $\mathrm{V}_{\mathrm{Ga}} \mathrm{O}_{\mathrm{N}}$ complex, where $\mathrm{V}_{\mathrm{Ga}}$ is the $\mathrm{Ga}$ vacancy. ${ }^{9}$ This assumption is based on the observation of different activation energies for thermal quenching of the YL band in undoped GaN samples ${ }^{12}$ and Si-doped GaN samples. We propose that this defect is the $\mathrm{V}_{\mathrm{Ga}} \mathrm{Si}_{\mathrm{Ga}}$ complex. Although it is predicted that the $\mathrm{V}_{\mathrm{Ga}} \mathrm{Si}_{\mathrm{Ga}}$ complex is not as stable as the $\mathrm{V}_{\mathrm{Ga}} \mathrm{O}_{\mathrm{N}}$ complex in $\mathrm{GaN},{ }^{22}$ the very high concentration of the $\mathrm{Si}_{\mathrm{Ga}}$ donors may lead to significant concentrations of this defect. The situation appears to be very similar to the $1.2 \mathrm{eV}$ band in GaAs which is attributed to the $\mathrm{V}_{\mathrm{Ga}} \mathrm{D}$ complexes with $\mathrm{D}$ being the shallow donors substituting for the $\mathrm{Ga}$ site $\left(\mathrm{Si}_{\mathrm{Ga}}, \mathrm{Ge}_{\mathrm{Ga}}\right.$, and $\left.\mathrm{Sn}_{\mathrm{GA}}\right)$ or As site $\left(\mathrm{Te}_{\mathrm{As}}\right.$, $\left.\mathrm{S}_{\mathrm{As}}, \mathrm{Se}_{\mathrm{As}}\right) \cdot{ }^{23,24}$ Similar to the $\mathrm{V}_{\mathrm{Ga}} \mathrm{D}$ complexes in $\mathrm{GaAs}$, it is expected that the electronic structure of the Ga vacancy in $\mathrm{GaN}$ dominates the electronic structure of the $\mathrm{V}_{\mathrm{Ga}} \mathrm{D}$ complexes involving a shallow donor. ${ }^{22}$

The very high IQE of PL observed in this work does not contradict the relatively high density of threading dislocations, on the order of $10^{9} \mathrm{~cm}^{-2}$, expected for GaN layers grown on sapphire by MOVPE. While the dislocations are commonly considered as the main source of nonradiative recombination in $\mathrm{GaN}$, the $\mathrm{Zn}$ acceptors with concentration above $10^{18} \mathrm{~cm}^{-3}$ and hole-capture cross-section of $\sim 10^{-13}$ $\mathrm{cm}^{2}$ (Ref. 9), can successfully compete with the nonradiative channel for the capture of minority carriers (holes), provided that the doping does not create point nonradiative defects in large numbers.

\section{SUMMARY}

We have fabricated high quality MOVPE GaN layers co-doped with silicon and zinc and have used several approaches to determine the absolute IQE of PL. Namely, (i) the characteristic temperature $T_{0}$ at which the thermal quenching of the BL band begins; (ii) the value of the increase in intensity of the YL and NBE bands caused by the quenching of the BL band; and (iii) the value of the rise of the relative IQE for the exciton band caused by a decrease of the BL band efficiency at high excitation intensities. With these independent approaches, we have estimated the absolute IQE of the BL band as $\eta_{10}=0.81-0.96$ in several GaN:Si,Zn samples at $T<250 \mathrm{~K}$ and low excitation intensity. The methods used here are very accurate when the IQE approaches unity since the size of the effect (e.g., an increase of the PL intensity for all of the PL bands caused by a quenching of a PL band with a high IQE) is very large compared to the errors in modeling the $I_{i 0}^{P L}(T)$ or $I_{i 0}^{P L}\left(P_{0}\right)$ dependencies. We have also determined the concentration of free electrons and estimated the concentrations of the $\mathrm{Zn}_{\mathrm{Ga}}$ and $\mathrm{Si}_{\mathrm{Ga}}$ centers in these samples from the PL measurements.

\section{ACKNOWLEDGMENTS}

The work was supported by the BMBF under the project "Ephquam." The authors also thank V. Avrutin and H. Morkoç from VCU for assistance with the Hall effect measurements.

${ }^{1}$ M. A. Reshchikov, Internal Quantum Efficiency of Photoluminescence in Wide-Bandgap Semiconductors, Chapter in Photoluminescence: Applications, Types and Efficacy, edited by M. A. Case and B. C. Stout (Nova Science Publishers, Inc., New York, 2012), pp. 53-120, ISBN: 978-1-61942426-5.

${ }^{2}$ H. Y. Ryu, K. H. Ha, J. H. Chae, K. S. Kim, J. S. Son, O. H. Nam, Y. J. Park, and J. I. Shim, Appl. Phys. Lett. 89, 171106 (2006).

${ }^{3}$ Q. Dai, M. F. Schubert, M. H. Kim, J. K. Kim, E. F. Schubert, D. D. Koleske, M. H. Crawford, S. R. Lee, A. J. Fischer, G. Thaler, and M. A. Banas, Appl. Phys. Lett. 94, 111109 (2009).

${ }^{4}$ X. Ni, J. Lee, M. Wu, X. Li, R. Shimada, Ü. Özgür, A. A. Baski, H. Morkoç, T. Paskova, G. Mulholland, and K. R. Evans, Appl. Phys. Lett. 95, 101106 (2009).

${ }^{5}$ H. Yoshida, M. Kuwabara, Y. Yamashita, K. Uchiyama, and H. Kan, Appl. Phys. Lett. 96, 211122 (2010).

${ }^{6}$ M. A. Reshchikov, A. G. Willyard, A. Behrends, A. Bakin, and A. Waag, Appl. Phys. Lett. 99, 171107 (2011).

${ }^{7}$ D. C. Look and R. J. Molnar, Appl. Phys. Lett. 70, 3377 (1997).

${ }^{8}$ J. D. McNamara, M. Foussekis, H. Liu, H. Morkoç, M. A. Reshchikov, and A. A. Baski, "Temperature Dependent Behavior of the SPV for n-type GaN,” Proc. SPIE 8262, 826213 (2012).

${ }^{9}$ M. A. Reshchikov and H. Morkoç, J. Appl. Phys. 97, 061301 (2005).

${ }^{10}$ M. A. Reshchikov, A. Kvasov, T. McMullen, M. F. Bishop, A. Usikov, V. Soukhoveev, and V. A. Dmitriev, Phys. Rev. B 84, 075212 (2011).

${ }^{11}$ M. A. Reshchikov, H. Morkoç, R. J. Molnar, D. Tsvetkov, and V. Dmitriev, Mater. Res. Soc. Symp. Proc. 743, L11.1 (2003).

${ }^{12}$ M. A. Reshchikov and R. Y. Korotkov, Phys. Rev. B 64, 115205 (2001).

${ }^{13}$ R. Y. Korotkov, M. A. Reshchikov, and B. W. Wessels, Physica B 325, 1-7 (2003). 
${ }^{14}$ W. Shockley and J. W. T. Read, Phys. Rev. 87, 835 (1952).

${ }^{15}$ V. N. Abakumov, V. I. Perel, and I. N. Yassievich, Nonradiative Recombinations in Semiconductors (Elsevier, Amsterdam, 1991).

${ }^{16}$ A. Dmitriev and A. Oruzheinikov, J. Appl. Phys. 86, 3241 (1999).

${ }^{17}$ M. A. Reshchikov, Appl. Phys. Lett. 88, 202104 (2006).

${ }^{18}$ J. R. Taylor, An Introduction to Error Analysis, second ed. (University Science Books, Sausalito, CA, 1997).

${ }^{19}$ T. Malinauskas, K. Jaračiūnas, R. Aleksiejunas, D. Gogova, B. Monemar, B. Beamont, and P. Gibart, Phys. Status Solidi B 243, 1426 (2006).
${ }^{20}$ J. S. Im, A. Moritz, F. Steuber, V. Härle, F. Scholz, and A. Hangleiter, Appl. Phys. Lett. 70, 631 (1997).

${ }^{21} \mathrm{O}$. Brandt, H.-J. Wünsche, H. Yang, R. Klann, J. R. Müllhäuser, and K. H. Ploog, J. Cryst. Growth 189-190, 790 (1998).

${ }^{22}$ J. Neugebauer and C. G. Van de Walle, Appl. Phys. Lett. 69, 503 (1996).

${ }^{23}$ E. W. Williams, Phys. Rev. 168, 922 (1968).

${ }^{24}$ N. S. Averkiev, A. A. Gutkin, E. B. Osipov, M. A. Reshchikov, and V. R. Sosnovskii, Defect Diffus. Forum 103-105, 31 (1993). 